\title{
Correction to: Cost Effectiveness of Introducing Etonorgestrel Contraceptive Implant into India's Current Family Welfare Programme
}

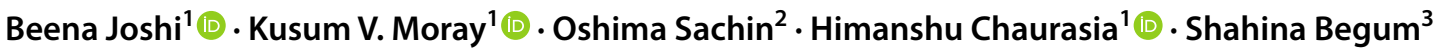

Published online: 27 October 2020

(c) Springer Nature Switzerland AG 2020

\section{Correction to: Applied Health Economics and Health Policy https://doi.org/10.1007/s40258-020-00605-5}

Unfortunately the Code availability statement has been published incorrectly in the original version of the article.

Previously read as:

"The coding used in the model is available in ESM."

Should read as:

"Will be made available on request."

The Electronic Supplementary Material (ESM) containing the model code has been removed.

The original article has been corrected.

The original article can be found online at https://doi.org/10.1007/ s40258-020-00605-5.

Beena Joshi

bjoshithane@gmail.com

1 Health Technology Assessment Resource Hub, Indian Council of Medical Research, National Institute for Research in Reproductive Health, Jehangir Merwanji Street, Parel, Mumbai 400012, India

2 Department of Health Research, Ministry of Health and Family Welfare, New Delhi, India

3 Biostatistics Department, Indian Council of Medical Research, National Institute for Research in Reproductive Health, Jehangir Merwanji Street, Parel, Mumbai 400012, India 